\title{
Analysis of Influential Factors for Investors' Stock Fund Performance in China
}

\author{
Xu Lingjuan Zhang Guoli Li Qian
}

\author{
College of Economics and Management \\ Nanjing University of Aeronautics and Astronautics, China \\ Email:Linda_xu@nuaa.edu.cn
}

\author{
Doi:10.5901/ajis.2013.v2n11p81
}

Abstract

Stock fund is an important species in China's mutual fund market. This paper takes stock fund investors as research object, through sample survey, works out the main influential factors of stock fund performance by principal component analysis (PCA). The result shows that International macro-environmental, stock fund management efficiency and investment strategies, policies and regulations regulatory, monetary policy, investors characteristics, scale cost are six major factors for the performance of investors' stock fund. In the end, we propose some suggestions to improve the performance.

Keywords: Stock fund, Investment performance, Principal component analysis, Influential factors

\section{Introduction}

Stock fund is one of the most important mutual funds in China, holding a leading role in China's mutual fund market as well as the capital market. Statistics show that by the end of 2012 China's stock funds has a net value of 1978.756 billion Yuan, with about 470 billion Yuan in absolute revenue, gaining $7.2 \%$ annualized excess return comparing the market base ${ }^{[1]}$. Therefore, it's drawing more and more attention.

With the expanding of the number and scale of stock fund, it has become a question for medium and small investors with little professional knowledge and experience to choose the most suitable product. For the fund management companies, it's also a problem to improve their revenue and scientifically assess managers' performance as well as create a fairly competitive environment. As to regulators, how to make surveillance and regulative rules to effectively protect investors' rights is also question that worth thinking.

\section{The Definition of Stock Fund Performance and Necessity of Studying the influential Factors}

Investors' stock fund performance refers to the real revenue investors get from stock fund[2]. It can be affected by macroenvironment, the ability of fund management companies and investors' own choices. Investors' stock fund performance can not only shows the ability of fund managers, but also reflects the situation of macro economy[3][4].

To study the influential factors for stock fund performance is important in the following ways[5][6]: Firstly, for medium and small investors with a limited possession of professional knowledge and ability to get and analyze information, it would provide a full-range of the performance, so they can make choices according to their different needs for riskrevenue liquidity and market characteristics in order to avoid the loss caused by blind follow. Secondly, from the fund management companies' prospective, they can better decide whether a strategy is suitable in the market and can assess the ability of managers, so as to create an outer restraint and motivation mechanism. Thirdly, from capital market regulators' prospective, through assessing the performance of different type and style of stock fund, they can have a better knowledge of the fund's features and improve their efficiency when examine and approve new funds, so as to secure a balanced development of fund market.

\section{The Design, Issuance and Collection of Questionnaire}

\subsection{The Design and Mark Scheme of Questionnaire}

Our study adopted face-to-face interview and Questionnaire Star online survey as two main methods. In the former one, we interviewed over twenty fund investors to get brief grasp of their ability to undertake risks, their knowledge about stock 
funds and the proportion of funds in domestic equity, the profitability of stock funds and their investment strategy.

After the interview, we designed a questionnaire on the influential factors of China's investors' stock fund performance based on the research done by scholars from home and abroad. There are ten quiz in four types as Single Choice, Logic-jump, Matrix-scale and Sorting. The content includes basic information of stock fund investment, macro environment that affects stock funds, macro policies, capital market fund characteristics, investors' characteristics, fund management companies and managerial teams. To ensure the effectiveness of questionnaire, we conducted an informal test first among financial managers from five banks and three securities companies as well as the above-mentioned over twenty investors. After that we delete highly-relative items as exchange rate fluctuation, and also delete those with bad response like investors' education level, stamp duty, fiscal investment plan and stock index futures. We also made modification and adjustment to those not clearly-explained items like profitability of funds in order to get the final edition of the questionnaire.

Table 1: Influential Factors for Investors' Stock Fund Performance

\begin{tabular}{|c|l|c|l|}
\hline No. & Influential Factors & No. & Influential Factors \\
\hline 1 & Change in balance of payment & 12 & market risk \\
2 & Fluctuations in global economy & 13 & Investment intensity of Top 10 stocks \\
3 & Change in benchmark rate & 14 & Turnover rate \\
4 & Change in reserves rate & 15 & Final asset value \\
5 & Policies to support industry & 16 & Favorable terms for purchase \& redeem \\
6 & Publication of securities law & 17 & Investors' income level \\
7 & Government's intervention & 18 & Time length of investment \\
8 & Expectation of market & 19 & Ability and attitude to take risks \\
9 & Large shareholders \& insider dealing & 20 & Punished expanse of redeem \\
10 & Expansion of market & 21 & Performance of the fund company \\
11 & Price fluctuation of large items & 22 & Stability of managerial team \\
\hline
\end{tabular}

In the formal questionnaire, we listed 22 factors concerning investors' stock fund performance in China (Table 1), and asked financial experts and investors to choose, judge and fill in with their real feelings. Then we assess the effectiveness of our survey.

\subsection{The Issuance and Collection of Questionnaire}

We adopt customizable link to find financial experts and university professors to fill in this questionnaire. At the same time, we found our sample through Questionnaire Star, a famous online survey website to further conduct the survey. To ensure the effectiveness of data collected, our object is those who had already filled and willing to continue filling the quiz. People from the same IP address can only participate once. We also have time control and artificial screen through the whole process to get the update. By strict examination, we collected 169 questionnaires in all, 121 of them is valid, counting $71.59 \%$ of the total.

\section{Principal Component Analysis of Influential Factors of Investors' Stock Fund Performance}

\subsection{Brief Introduction of PCA}

Principal Component Analysis (PCA) was first proposed by Hotelling in 1933. It uses the thought of dimension reduction to make large number of factors to compound factors while minimize information loss. The compound factors are referred to as principal components, whose number is far less than original factors. Every principal is the linear combination of original factors, and independent from each other ${ }^{[7]}$.

\subsection{Empirical Study}

To make the result scientific, we use SPSS19.0 software to analyze data ${ }^{[8]}$. Firstly, we verify the feasibility of PCA through Bartlett test of sphericity and KMO test. The purpose of KMO test is to analyze simple correlation ratio and partial correlation ratio and find out whether PCA can be adopted. Input 22 influential factors and their mark into SPSS 19.0, 
select Factor Analysis and check Principal Component Analysis, we can get the following KMO and Bartlett Table (Table 2).

Table 2: KMO and Bartlett Test

\begin{tabular}{|l|c|c|}
\hline Kaiser-Meyer-Olkin Measure of Sampling Adequacy. & .762 \\
\hline \multirow{3}{*}{ Bartlett's Test of Sphericity } & Approx. Chi-Square & $1,279.285$ \\
\cline { 2 - 3 } & $\mathrm{df}$ & 301 \\
\cline { 2 - 3 } & Sig. & .000 \\
\hline
\end{tabular}

According to Kaiser's KMO standard, the result 0.762 is larger than 0.5 , making it suitable foe PCA. Bartlett test of sphericity is 1279.285 with the significance level $\mathrm{P}(\mathrm{sig}=0.000)<0.05$, denying original hypothesis $\mathrm{HO}$ (Variables are unrelated.). Therefore, the result is statistically significant.

Second, use PCA to analysis the data collected, choose those with characteristic value higher than 1 and get Total Variance Explained (Table 3). The table shows statistics information of principal components, including squared arraying from large to small, each component's variance contribution rate and cumulative rate.

Finally, work out the number of principal components. It can be seen from the table that there are 6 components with characteristic value larger than 1 , their cumulative variance contribution is $70.092 \%$ of the total variance, representing most of the information. Through max variance rotation, we array those factors with higher load together under the same component to get the Principal Component Loading Matrix. In this way, the original 22 factors have been reduced to 6 principal components, the dimension reduction is achieved.

Table 3: Total Variance Explained

\begin{tabular}{|c|c|c|c|c|c|c|}
\hline \multirow{2}{*}{ Component } & \multicolumn{3}{|c|}{ Initial Eigenvalues } & \multicolumn{3}{c|}{ Extraction Sums of Squared Loadings } \\
\cline { 2 - 7 } & Total & $\%$ of Var & Cum \% & Total & \% of Var & Cum\% \\
\hline 1 & 5.273 & 22.720 & 22.720 & 5.273 & 22.720 & 22.720 \\
2 & 3.121 & 13.552 & 36.272 & 3.121 & 13.552 & 36.272 \\
3 & 2.824 & 11.291 & 47.563 & 2.824 & 11.291 & 47.563 \\
4 & 1.971 & 8.681 & 56.244 & 1.971 & 8.681 & 56.244 \\
5 & 1.826 & 7.026 & 63.270 & 1.826 & 7.026 & 63.270 \\
6 & 1.541 & 6.822 & 70.092 & 1.541 & 6.822 & 70.092 \\
7 & .997 & 3.115 & 73.207 & & & \\
8 & .879 & 3.094 & 76.301 & & & \\
9 & .845 & 2.740 & 79.041 & & & \\
10 & .655 & 2.577 & 81.618 & & & \\
11 & .624 & 2.237 & 83.855 & & & \\
12 & .571 & 2.225 & 86.080 & & & \\
13 & .476 & 2.005 & 88.085 & & & \\
14 & .433 & 1.868 & 89.953 & & & \\
15 & .389 & 1.774 & 91.727 & & & \\
16 & .339 & 1.543 & 93.270 & & & \\
17 & .322 & 1.463 & 94.733 & & & \\
18 & .304 & 1.381 & 96.114 & & & \\
19 & .267 & 1.213 & 97.327 & & & \\
20 & .257 & 1.168 & 98.495 & & & \\
21 & .189 & 0.859 & 99.354 & & & \\
22 & .022 & 0.646 & 100.000 & & & \\
\hline
\end{tabular}

\subsection{Naming of Principal Components}

According to Table 4.4, we also find out that the 22 influential factors under a certain principal component all have higher load and a clear structure, this means our questionnaire has a pretty good design.

Under Component Z1, Fluctuations in global economy, Change in balance of payment, Price fluctuation of large items, market risks and expansion of market have higher load, contributing $22.720 \%$. Those factors reflect the influence 
of international environment change on the whole, so we can call it External International Macro Environment Component.

Under Component Z2, Turnover rate, Performance of the fund company, Investment intensity of Top 10 stocks and Stability of managerial team have higher load, contributing $13.552 \%$ of the total. Those factors reflect the systematic stability of manager, asset allocation, investment style and operational risk, so we can call it Management Efficiency and Investment Strategy Component.

Under Component Z3, Government's intervention, Publication of securities law, large shareholders \& insider dealing, Policies to support industry have higher load, contributing $11.291 \%$ of the total. Those factors show that under government's intervention and industrial policies, the performance of stock fund has a close relation with the blue chips, so we can call it Policy Regulative Component.

Under Component Z4, Government's intervention, benchmark rate and reserve rate made by Central Bank have higher load. China 's security market is an emotion-motivated one, positive monetary policies will boost public ' $\mathrm{s}$ confidence in future economy, so stock fund will also fluctuate with security market. We can call it Monetary Policy Component.

Table 4: Orthogonal Rotation Component Matrix

\begin{tabular}{|l|c|c|c|c|c|c|}
\hline \multirow{2}{*}{\multicolumn{1}{|c|}{ Influential Factors }} & \multicolumn{6}{|c|}{ Principal Component } \\
\cline { 2 - 7 } & Z1 & Z2 & Z3 & Z4 & Z5 & Z6 \\
\hline Fluctuations in global economy & .747 & .210 & -.024 & .023 & -.209 & -.134 \\
Price fluctuation of large items & .712 & .312 & .173 & .259 & -.121 & .187 \\
Change in balance of payment & .700 & .272 & .068 & .012 & .025 & .040 \\
Risks in securities market & .673 & .197 & .381 & .049 & .203 & .357 \\
Expansion of market & .587 & .073 & .168 & .433 & .291 & .122 \\
Turnover rate & .149 & .766 & .041 & -.118 & .111 & .208 \\
Performance of the fund company & -.043 & .683 & .114 & .038 & .208 & .137 \\
Investment intensity of Top 10 stocks & -.272 & .640 & .437 & .723 & .375 & .194 \\
Stability of managerial team & -.411 & .562 & .419 & .707 & .089 & -.128 \\
Government's intervention & .237 & -.121 & .801 & .656 & -.113 & -.003 \\
Publication of securities law & .048 & -.361 & .718 & -.696 & .221 & .350 \\
Large shareholders \& insider dealing & -.379 & -.176 & .676 & .302 & .423 & .223 \\
Policies to support industry & .275 & .318 & .519 & .471 & -.310 & -.127 \\
Change in benchmark rate & .372 & -.414 & .414 & .845 & .086 & -.019 \\
Change in reserves rate & .216 & .077 & .307 & .693 & .339 & .116 \\
Ability and attitude to take risks & -.012 & .432 & .432 & .297 & .867 & -.017 \\
Time length of investment & .194 & .165 & .165 & -.031 & .801 & -.623 \\
Expectation of market & .144 & -.278 & -.278 & .315 & .704 & -.815 \\
Investors' income level & -.012 & .132 & .132 & -.393 & .662 & .407 \\
Favorable terms for purchase \& redeem & .210 & .345 & .345 & .538 & .083 & .804 \\
Punished expanse of redeem & .276 & .424 & .424 & .318 & .279 & .785 \\
Final asset value & .358 & -.117 & -.117 & .226 & .195 & .714 \\
\hline Method: PCA & \multicolumn{7}{|c|}{} \\
Method of rotation: Orthogonal Rotation with Kaiser standard \\
a. rotation convergent after 9 iterations
\end{tabular}

Under Component Z5, Ability and attitude to take risks, Time length of investment, Expectation of market, Investors' income level have higher load. Those reflect investors' personal characteristics like investment idea and risk preference and income. We can call it Investors' Characteristics Component.

Under Component Z6, Favorable terms for purchase \& redeem, Punished expanse of redeem, Final asset value have higher load. Those reflect investors' purchase and redeem cost and fund scale. We name it Scale Cost Component.

Table 5: Naming of Principal Components

\begin{tabular}{|ccccccc|}
\hline Principal Components & Z1 & Z2 & Z3 & Z4 & Z5 & Z6 \\
\hline \multirow{2}{*}{ Name } & External & Management & Policy Regulative & Monetary & Investors' & Scale Cost \\
& International & Efficiency and & Component & Policy & Characteristics & Component \\
\hline
\end{tabular}




\begin{tabular}{|ccccccc|}
\hline & Macro & Investment & & Component & Component \\
& $\begin{array}{c}\text { Environment } \\
\text { Component }\end{array}$ & $\begin{array}{c}\text { Strategy } \\
\text { Component }\end{array}$ & & & & \\
Total & 5.273 & 3.121 & 2.824 & 1.971 & 1.826 & 1.541 \\
\% of Variance & 22.720 & 13.552 & 11.291 & 8.681 & 7.026 & 6.822 \\
Cumulative \% & 22.720 & 36.272 & 47.563 & 56.244 & 63.270 & 70.092 \\
\hline
\end{tabular}

\subsection{Analysis of Effectiveness}

The Analysis of effectiveness includes reliability and validity. Our research uses half- reliability as the reliability indicator of questionnaire. To get this, we divide even or odd items by half, work out their relative ratio $r_{x x}$, then calculate the reliability index $R_{X X}$ for the whole questionnaire according to Spearman-Brown Formula. The Formula is: $R_{X X}=2 r_{x x} /\left(1+r_{x x}\right)$. The reliability index we get is 0.862 , which is acceptable, showing that our result is reliable.

Validity refers to the correlation between result and a certain external standard. The methods include content validity, estimation validity, idea validity, polymerization validity and differentiation validity. Our research adopts content validity $(\mathrm{CV})$ to assess the validity of questionnaire. The formula is:

$$
C V=\left(n_{e}-\frac{n}{2}\right) / \frac{n}{2}
$$

In the formula, $n_{e}$ refers to the number of people who think items can ideally reflect content; $n$ is the total number of people. To ensure its validity, that is, the correlation between 22 items and the content, we conducted validity test on the 121 effective questionnaires collected. Result shows that 102 out of 121 think the questionnaire can ideally reflect the influential factors for investors' stock fund performance in China with the figure CV is 0.843 . It means the result is valid.

\section{Conclusion and Suggestion}

Based on current research result, we designed the questionnaire and conducted the survey on 207 specific samples by interview and Questionnaire Star online survey and found out six principal component of Investors' stock fund performance in China named Global Macro Environment Component, Management Efficiency and Investment Strategy Component, Policy Regulative Component, Monetary Policy Component, Investors' Characteristics Component and Scale Cost Component.

According to the above, if investors and fund management companies want to improve the performance of investors' stock fund in China, and the regulators want to protect investment rights of medium and small investors, they should consider these six principal components thoroughly, especially the two key components as Global Macro Environment Component and Management Efficiency and Investment Strategy Component.

As for medium and small investors, they should understand and have the knowledge of the consistency of the performance of stock fund and macro economy cycle ${ }^{[9]}$. Choose and shift their funds according to macro economy's operational region. Concretely speaking, they may choose stock fund or index fund during economy recovery and bloom because those can take full advantage of the bull market. When the economy is too hot, they can choose large item fund and during inflation, the monetary fund will be best choice. In the end, bond fund and capital preservation fund will be preferred in a recession. In strategy, investors should shake off the idea of leaving everything to fund managers or companies, take active measures to find the most suitable funds for themselves. Besides, with the high systematic risk and low market efficiency, markets tend to rise and fall in the same direction, so it's important to make allocations in assets with different level of risks when choosing investment objects. Putting all the money in stock fund is not wise.

As for fund managers, fund management efficiency and investment strategy is of vital importance in deciding the performance of investors' stock fund, contributing $13.552 \%$ in all. Therefore, fund managers should improve the performance in the following ways: firstly, enhance the study on macro market trend. Secondly, have all-range training on investors about rational investment. Thirdly, make innovations and create more types of funds with different risk levels, investment styles and reasonable scales. Finally, establish indicator system to assess the performance of stock fund and adjust their strategy in time according to the assessment.

As for regulators, a win-win result between investors, listed companies and fund management companies can only be achieved by safeguarding a good order in securities market, improving the market efficiency and protect the rights of investors. Therefore, regulative department should shift the emphasis to the regulation of fund management companies, 
education and protection of investors, the fairness of market competition and full disclosure of information rather than the role of developing the fund industry.

\section{Acknowledgement}

This study was supported by the basic research funding of Nanjing University of Aeronautics and Astronautics, as a special research focus project (NR2013007).

\section{Reference}

Galaxy Securities. Make idle money move, invest in monetary fund and sup-short bond fund.[DB/OL]. http://www.cnfund.cn/news/news/2011/12/26/820111226288349.html

Wang Cong. Analysis on Assessment Model of Securities Fund [J].Economic Research, 2001(9):31-38.

Treynor Jack. How to Rate Management of Investment Funds[]]. Harvard Business Review,1965(1):63-75.

William F,Sharp. Mutual Fund Performance[J].Journal of Bushiness,1966(39): 119-138.

Wu Shuang. Study on the Performance of Open-end Fund in China. [D].Wuhan: South China University of Technology,2010.

Wang Maoling. Appraisal and Empirical Study on Performance of stock open-end fund in China. [D].Beijing: Minzu University of China,2010.

Wang Lu. Basic ideas, application and practice for SPSS [M].1st Edition, Beijing: Chemistry and Chemical Technology Press,2010. 175$176,218-229$.

Xue Wei.Statistic Analysis and the Application of SPSS [M].Beijing: China Renmin University Press,2011,321-339.

Liu Lanbiao, Gu Jingjing, Li Baowei. The Basic Relationship between China;s Securities Market and Economic Fluctuations. [J].Nankai Economic Review, 2001(3):57-62. 